\title{
Determinantes de la productividad laboral en México: una aproximación desde la teoría del crecimiento endógeno con redes neuronales artificiales
}

\author{
Héctor Eduardo Díaz Rodríguez y Fidel Aroche Reyes
}

\section{Resumen}

La adopción masiva de TIC ha incrementado la investigación sobre el vínculo entre estas y el crecimiento económico. Existe un impacto significativo del uso de TIC sobre el crecimiento, pero los estudios tienden a ignorar los determinantes del uso de tales tecnologías y las condiciones diferenciadas de las empresas, industrias y países que las adoptan. Aquí se analizan los determinantes del impacto diferenciado de las TIC sobre el crecimiento de la productividad laboral en las empresas mexicanas, utilizando microdatos de la Encuesta sobre Tecnologías de la Información y las Comunicaciones (ENTIC) del Instituto Nacional de Estadística y Geografía (INEGI); se aplican técnicas de análisis factorial y de redes neuronales artificiales. Los resultados indican que la fortaleza del vínculo con el sector externo y las capacidades (nivel educativo y madurez organizacional) determinan el impacto de las TIC en la productividad laboral de México.

\section{Palabras clave}

Empleo, trabajo, productividad del trabajo, innovaciones, tecnología de la información, tecnología de las comunicaciones, redes de computadoras, México

Clasificación JEL

$\mathrm{C} 46, \mathrm{O} 47, \mathrm{O} 33$

\section{Autores}

Héctor Eduardo Díaz Rodríguez es Profesor y Jefe de la Licenciatura en la División de Estudios Profesionales de la Facultad de Economía de la Universidad Nacional Autónoma de México (UNAM). Correo electrónico: hectoreduardo12@comunidad.unam.mx.

Fidel Aroche Reyes es Profesor en la División de Estudios de Posgrado de la Universidad Nacional Autónoma de México (UNAM). Correo electrónico: aroche@unam.mx. 


\section{Introducción}

En los últimos 20 años ha habido un auge de los estudios acerca del impacto de la adopción de tecnologías en todo el mundo, a la par de la importancia que ellas han adquirido en los procesos productivos, en particular las tecnologías de la información y las comunicaciones (TIC). La proliferación de dichos estudios comenzó poco después de la aparición de las teorías del crecimiento endógeno (los conocidos modelos $A K$ ) en la década de 1990, que resaltan que, junto con la tecnología, la inversión en capital humano es una fuente importante de crecimiento de la productividad; paradójicamente, sin embargo, la mayoría de los análisis tempranos sobre el impacto de las TIC dejaron de lado la importancia de la educación y la capacitación de la fuerza de trabajo como factor determinante del aprovechamiento tecnológico (Brynjolfsson, 1993 y 1996; Brynjolfsson y Hitt, 1996; Bresnahan, 1997 y 1999; Lichtenberg, 1995; Jorgenson y Stiroh, 2000). Es decir, la innovación tecnológica y de productos ha tomado el papel más relevante en esta perspectiva.

Solo en los años recientes algunos estudios han reconocido que las condiciones iniciales diferenciadas que enfrentan los distintos agentes (empresas, industrias o países) son importantes para entender la relación entre las TIC y la productividad; no obstante, estos estudios son todavía pocos en relación con los existentes en el tema y se preocupan principalmente por las economías desarrolladas (Schreyer, 2000; Daveri, 2000; Dutta y Bilbao-Osorio, 2012; Bilbao-Osorio, Dutta y Lanvin, 2013; UIT/ UNESCO, 2013). Los estudios sobre el impacto de las TIC en las economías en desarrollo han sido, en el mejor escenario, escasos y prácticamente no se ha considerado el papel que la educación desempeña en aquel vínculo, como queda de manifiesto en los pocos estudios que analizan el papel de las TIC y su impacto en la productividad en México (Aravena, Cavada y Mulder, 2012; INEGI, 2013; UNCTAD, 2003). Por lo anterior, el presente estudio busca aportar a la discusión acerca del papel que la educación y la capacitación de la fuerza de trabajo tienen como factores determinantes del aprovechamiento de la tecnología en México, retomando algunas ideas de la teoría del crecimiento endógeno. Para ello, se realiza un análisis de microdatos, con información proveniente de la Encuesta sobre Tecnologías de la Información y las Comunicaciones (ENTIC) del INEGI, mediante las metodologías de análisis factorial y de redes neuronales artificiales (Haykin, 1999; Arrieta, Torres y Velásquez, 2009; Pitarque, Roy y Ruiz, 1998; Larrañaga, Inza y Moujahid, 2003).

La hipótesis es que, si bien las TIC representan una condición necesaria para conseguir el crecimiento de la productividad laboral, por sí solas son insuficientes; para que dicho crecimiento ocurra, se requiere que las organizaciones y los trabajadores cuenten con un conjunto de capacidades que les posibiliten incorporar la tecnología dentro de los procesos productivos y de negocios, y potenciar su aprovechamiento. Sin la existencia de esas capacidades, la disponibilidad de las TIC se torna estéril.

El resto del artículo se estructura de la siguiente manera: en la segunda sección se revisan los estudios que analizan el impacto de las TIC en la productividad, a fin de describir algunos modelos originados en la teoría del crecimiento endógeno; en la tercera sección se describen las metodologías de análisis factorial y redes neuronales artificiales y en la cuarta se presentan y analizan los resultados de la aplicación del método a los datos de la encuesta; por último, se presentan las conclusiones y posibles opciones de política pública.

\section{Revisión de la literatura}

El conocido modelo de Solow (1957) es una de las primeras aproximaciones al impacto del cambio tecnológico y del crecimiento de los factores sobre la productividad, mediante una función de producción Cobb-Douglas. Esta mide la eficiencia con la que esos factores se emplean en el proceso de producción; 
así, la productividad puede crecer por dos vías: por un lado, por un incremento de la relación de capital por trabajador y, por el otro, por el uso de tecnología, que además de profundizar aquella relación, genera un uso más eficiente de la combinación de factores. Sin embargo, el modelo no explica el cambio técnico, ni las causas de un mejor aprovechamiento del mismo. Dadas las características específicas del modelo de Solow, se convertiría en el modelo por excelencia a partir del cual estudios posteriores analizarían el vínculo entre TIC y productividad¹.

Desde principios de la década de 1990, dada la incapacidad del modelo de Solow para explicar las fuentes del cambio técnico, surge una nueva oleada de estudios que, basados en aquel, incorporan el papel de la inversión en educación como fuente aceleradora del progreso técnico (Romer, 1990 y 1994; Aghion y otros, 1998).

Las primeras investigaciones sobre el papel de la adopción de computadoras en la productividad en la economía de los Estados Unidos no encontraron una conexión directa. Ello dio origen a la llamada "paradoja de la productividad", que estimuló nuevas investigaciones (tanto con series de datos más largas y confiables como con métodos más refinados), entre las que destacan las realizadas por Brynjolfsson (1993 y 1996), Brynjolfsson y Hitt (1996), Bresnahan (1997 y 1999), Lichtenberg (1995) y Jorgenson y Stiroh (2000). Este conjunto de estudios revela la existencia de un cambio importante en la productividad, generado por el uso de las TIC. Para diferenciar el impacto del uso exponencial de TIC en las organizaciones de la adopción de otro tipo de tecnología, esta serie de estudios (por lo demás, basados en el modelo de Solow) encontraron una diferencia fundamental entre las inversiones en TIC y otro tipo de capital, y es el doble papel que desempeñan las inversiones en TIC: primero, como cualquier otro tipo de capital, las TIC pueden ser consideradas como una nueva tecnología de producción que mejora la productividad laboral; segundo, pueden también considerarse un elemento transformador de los procesos productivos.

Un conjunto de investigaciones (Bresnahan, 1997; Gurbaxani y Whang, 1991; Malone, Yates y Benjamin, 1989; Hollenstein, 2004; Capel y Bosch, 2004; Inklaar, O'Mahony y Timmer, 2005; Bayo-Moriones y Lera-López, 2007; Carrera Portugal, 2010; Tödtling, Grillitsch y Höglinger, 2012; Peppard y Ward, 2016) han destacado que el impacto del segundo elemento es mayor, ya que induce cambios en los procesos de negocio, tanto dentro de las organizaciones como entre ellas. En este sentido, este conjunto de investigaciones atribuyen a las TIC el valor de potenciar cambios en los procesos de negocio y, con ellos, en la productividad multifactorial. Greenan, Mairesse y Topiol-Bensaid (2001) estudian el impacto de las TIC en algunas empresas francesas y encuentran un resultado similar al de las investigaciones de Bresnahan (1997) y Lichtenberg (1995).

En oposición a estos resultados, Lal (2001) no encuentra relación entre las inversiones en TIC y la productividad en la industria textil de la India; el contraste de los resultados entre los estudios realizados para empresas ubicadas en economías desarrolladas y en economías en desarrollo tiende a ser generalizado, como lo demuestran Kraemer y Dewan (2000) y Pohjola (2001), que encuentran información en el sentido de que, a nivel de empresa, las TIC tienden a generar cambios en la productividad en países desarrollados, pero no en países en desarrollo. Kraemer y Dewan (2000) y Pohjola (2001) encuentran como razón fundante de esas diferencias los costos de la mano de obra entre ambos grupos de países; en los países en desarrollo, los costos laborales tienden a ser más bajos y los costos de acceso a capital son altos, lo que hace relativamente más costosa la sustitución de trabajo por capital. Lo contrario ocurre en los países desarrollados².

1 Debido a que el modelo permite descomponer el crecimiento tanto en los factores que lo originan (capital y trabajo o un uso más eficiente de las combinaciones de ambos) como en el tipo de capital (inversiones en TIC o en aspectos distintos de las $\mathrm{TIC})$ que lo genera.

2 Desde el punto de vista del presente estudio, las diferencias del impacto de las TIC entre países y actividades económicas se explican más por los distintos niveles de educación de la mano de obra, el distinto grado de madurez de las empresas existente entre ambos grupos y la distinta composición sectorial. 
No obstante, la gran cantidad de estudios basados en el modelo de Solow no ha logrado explicar de manera satisfactoria el hecho de que, mientras la adopción de TIC en el mundo se ha incrementado de manera exponencial, la productividad y el crecimiento económico, que tendrían que ser resultado de la primera, muestran patrones tan distintos de evolución, la mayoría de las veces tendientes al estancamiento, como se ejemplifica de forma contundente con la economía mexicana. Debido a lo anterior, algunas investigaciones más recientes han comenzado a analizar factores relacionados con el contexto de las economías y empresas, así como con diferencias en sus condiciones iniciales, para tratar de explicar por qué niveles similares de adopción de TIC han producido resultados tan disímiles en términos de productividad. Entre estas últimas, los estudios de Aramendia-Muneta y Ollo-López (2013), Tarutè y Gatautis (2014) y una serie de análisis realizados por organismos de desarrollo y cooperación internacional (Banco Mundial, 2011 y 2012; Dutta y Bilbao-Osorio, 2012; Bilbao-Osorio, Dutta y Lanvin, 2013; OCDE, 2012) concluyen que si las TIC no han logrado generar impactos en economías o empresas dentro de ellas, se debe a la rigidez de factores institucionales que inhiben el aprovechamiento de sus potenciales beneficios. La conclusión de estos organismos es la misma de hace 30 años: se requiere la creación de condiciones generales para un crecimiento elevado y sostenido, a saber, apertura al comercio y a los flujos de capital, un marco institucional y legal adecuado, flexibilidad en la estructura productiva y mercados eficientes (Banco Mundial, 2011 y 2012; Dutta y Bilbao-Osorio, 2012; Billbao-Osorio, Dutta y Lanvin, 2013; OCDE, 2012).

Desde la perspectiva de este estudio, no existe un vínculo directo entre la disponibilidad de TIC y el desempeño económico, visto como cambio en la productividad de los factores, o el crecimiento agregado de la economía; ese vínculo es más bien potencial y depende de que las economías, industrias o empresas involucradas posean un conjunto de características y capacidades que hagan fructífero el uso de la infraestructura de TIC. La influencia de tales determinantes depende del momento, de la economía en particular de la que se trate, de la existencia de un conjunto de capacidades vinculadas al nivel educativo de la fuerza de trabajo y a la madurez de las organizaciones, traducida en capacidades para incorporar las TIC en sus procesos productivos e, incluso, de las propias características de las actividades económicas desarrolladas.

El modelo de Solow al que se hizo referencia y los estudios analizados en esta sección son incapaces de explicar las diferencias en la productividad derivadas de la adopción de TIC, si bien su materia de estudio se encuentra constituida principalmente por economías desarrolladas; en el caso de las economías en desarrollo, la necesidad de estudiar el tema desde una perspectiva distinta se hace más patente debido a la escasa investigación que existe. En el caso específico de la economía mexicana, la adopción masiva de TIC por parte de las empresas no ha generado un incremento de la productividad y resulta oportuno brindar posibles explicaciones al respecto.

\section{Metodología y características de la información}

Con el objetivo de entender las razones por las cuales la adopción de TIC y su uso creciente no han producido efectos positivos en la productividad en México, en este trabajo se analiza la base de datos provenientes de la Encuesta sobre Tecnologías de la Información y las Comunicaciones (ENTIC) del INEGI publicada en 2013, que recoge información de 389 variables sobre el uso de TIC en una muestra de 6.468 empresas, representativas de 157.611 organizaciones con diez empleados o más, clasificadas en 76 subsectores (según el Sistema de Clasificación Industrial de América del Norte (SCIAN)) durante el año anterior. A fin de aplicar el modelo que se presenta más adelante, se han seleccionado 34 variables, de las cuales 13 se emplean directamente y 21 mediante la construcción de dos índices, referidos al 
uso de Internet y de software, para medir la manera en que las organizaciones incorporan las TIC en sus procesos productivos y de negocios ${ }^{3}$.

Los criterios para agrupar las variables en estos índices fueron los siguientes: cada una de las variables disponibles sobre usos de Internet, de software y de la nube se ordena en función de las capacidades requeridas para su implementación; de acuerdo con este orden, se asigna una calificación ${ }^{4}$, menor para los usos catalogados como básicos ${ }^{5}$ y mayor para los avanzados. De esta forma, el cálculo permite reducir las 21 variables que componen los diversos usos de Internet y software a solo 2 variables: índice de usos de Internet e índice de usos de software. Estos índices se incluyen como variables para el análisis factorial.

\section{Análisis factorial}

El análisis factorial permite agrupar las variables correlacionadas y encontrar grupos homogéneos, particularmente en bases de datos con un gran número de series. En principio, estos grupos son independientes entre sí. El análisis factorial permite encontrar un mínimo de dimensiones capaces de explicar el máximo de información contenida en los datos. En otros términos, un factor es una dimensión cualitativa en un eje de coordenadas, que define la forma como las entidades (observaciones) difieren, así como el tamaño o el sabor de un objeto define sus dimensiones cualitativas (Domínguez y Brown, 2004).

Formalmente, el análisis factorial es un método multivariante que expresa $p$ variables observables como una combinación lineal de $m$ variables hipotéticas o latentes, denominadas factores. Estas variables o factores comunes se obtienen e interpretan a partir de la matriz de correlaciones entre las variables:

$$
R=\left(\begin{array}{cccc}
1 & r 12 & \cdots & r 1 p \\
r 21 & 1 & \cdots & r 2 p \\
\vdots & \vdots & \ddots & \vdots \\
r p 1 & \vdots & \cdots & 1
\end{array}\right)
$$

En el caso de los modelos multifactoriales las $p$ variables observables (variables de la ENTIC) dependen de $m$ variables latentes o factores comunes, además de $p$ factores únicos, de acuerdo con el siguiente modelo lineal:

\footnotetext{
3 Uso y aprovechamiento es un concepto que describe los usos que las organizaciones dan a la tecnología de la que disponen y la manera en que aprovechan sus ventajas. El concepto de uso no es lineal, en virtud de que existen diferentes niveles en los que el uso de la tecnología se incorpora dentro de los procesos productivos; a medida que los usos tienden a tener efectos en el desarrollo de procesos y generar cambios en las organizaciones, el impacto que tiene la tecnología tiende a ser mayor.

La clasificación propuesta aquí no busca asignar una calificación específica a cada uso, sino simplemente ordenarlos en función del grado de capacidades necesario para la puesta en práctica de cada uso específico.

4 Las variables que componen los índices de uso de Internet y de software, con los respectivos puntajes para su construcción, son las siguientes: en el caso del índice de usos de Internet: búsqueda de información general (4), transferencia de información (6), transacciones financieras (8), acceso a sitios gubernamentales (10), pago de servicios no gubernamentales (12), atención a proveedores (14), reclutamiento y selección de personal (16), servicio a clientes (18), publicidad de la empresa (20) y capacitación a distancia (22); en el caso del índice de usos de software: software para facturación (4), contabilidad (6), administración de nómina (8), administración de compras y pagos (10), recursos humanos (12), ventas (14), inventarios (16), administración del negocio (18), análisis de información (20), diseño (22) y logística (24).

5 Un uso se considera básico si las capacidades requeridas para ese uso son bajas; en la medida en que el uso es más complejo, se requiere de capacidades mayores para su implementación. Por ejemplo, la variable de búsqueda general de información, en el caso de Internet, requiere de pocas capacidades de los recursos humanos y las organizaciones. La variable de capacitación a distancia requiere de habilidades mayores, como el establecimiento de una cultura de capacitación, la infraestructura necesaria para realizarla y conocimiento de las herramientas tecnológicas por parte de la fuerza de trabajo, entre otras.
} 


$$
\begin{gathered}
X_{1}=a_{11} F_{1}+\cdots+a_{1 m} F_{m}+d_{1} U_{1} \\
X_{2}=a_{21} F_{1}+\cdots+a_{2 m} F_{m}+d_{2} U_{2} \\
\cdots \\
X_{p}=a_{p 1} F_{1}+\cdots+a_{p m} F_{m}+d_{p} U_{p}
\end{gathered}
$$

El modelo de análisis multifactorial parte de dos hipótesis fundamentales:

i) Los factores comunes y los factores únicos están incorrelacionados, es decir:

$$
\operatorname{Cor}\left(F_{i}, U_{j}\right) \quad i=1, \ldots, m \quad j=1, \ldots, p
$$

ii) Los factores comunes y los factores únicos tienen media 0 y varianza $1^{6}$ :

$$
\mu=0, \sigma=1
$$

En el modelo de análisis factorial, la varianza en su conjunto se explica por dos grupos de factores, por un lado, los factores comunes y, por otro, los factores únicos, como puede verificarse de acuerdo con lo siguiente:

$$
\operatorname{Var}\left(X_{i}\right)=a_{i 1}^{2}+\cdots+a_{i m}^{2}+d_{i}^{2}
$$

Es decir, $a_{i j}^{2}$ es la parte de la variabilidad de $X_{i}$ que se explica por la existencia de un factor común $\left(F_{j}\right)$, mientras que $d_{i}^{2}$ se interpreta como la parte que se explica exclusivamente por el factor único $U_{i}$.

La parte de la varianza conjunta de la variable $X$ explicada por factores comunes se denomina "comunalidad" y se obtiene a partir de la siguiente función:

$$
h_{i}^{2}=a_{i 1}^{2}+\cdots+a_{i m}^{2}
$$

\section{Redes neuronales artificiales}

Una red neuronal artificial es un procesador distribuido en paralelo de forma masiva con una propensión natural a almacenar conocimiento experimental y convertirlo en disponible para su uso (Haykin, 1999). Asemeja al cerebro en dos aspectos:

i) El conocimiento de una red neuronal artificial es adquirido, al igual que en el caso de una red neuronal biológica, mediante un proceso de aprendizaje.

ii) Las fuerzas de conexión entre las neuronas, denominadas ponderaciones sinápticas, se utilizan para almacenar el conocimiento generado mediante los procesos de aprendizaje.

El aprendizaje obtenido en una red neuronal artificial se emplea para estimar el comportamiento de una determinada variable, en función de variables con información de entrada; entre las variables de entrada (capa de entrada) y las variables de salida (capa de salida), existe un proceso en el que se estiman tanto la información como el modelo mediante el cual ella se analiza. Esta parte intermedia se conoce como "capa oculta".

\footnotetext{
6 Sin embargo, dado que los factores comunes representan dimensiones independientes en el sentido lineal y que tanto los factores comunes como los únicos son variables convencionales, es posible que se dé el caso de que los vectores independientes (comunes) no tengan media 0 y varianza 1 (Cuadras, 2007).
} 
Si bien un modelo de regresión puede considerarse un tipo específico de red, en el sentido de que los coeficientes de regresión pueden adquirir y almacenar conocimiento, la regresión tiene una estructura de modelo rígida, así como una estructura subyacente en los supuestos, que se imponen desde el inicio, a diferencia de lo que ocurre con un modelo de redes neuronales artificiales, en el que la forma de las relaciones se determina durante el proceso de aprendizaje. Si una relación lineal entre las variables dependientes e independientes es adecuada, los resultados de la red neuronal deben aproximarse lo máximo posible a los del modelo de regresión lineal. Si una relación no lineal es más adecuada, la red neuronal se aproximará automáticamente a la estructura "correcta" del modelo.

Una red neuronal artificial, de acuerdo con Larrañaga, Inza y Moujahid (2003), se puede definir como un grafo dirigido que cumple con las siguientes propiedades:

i) A cada nodo (neurona) $i$ se le asocia una variable de estado $X_{i}$.

ii) A cada conexión $(i, j)$ entre los nodos (neuronas) $i$ y $j$ se le asocia un peso $w_{i j} \in \mathbb{R}$.

iii) A cada nodo (neurona) $i$ se le asocia un umbral $\theta_{i} \in \mathbb{R}$.

iv) Para cada nodo $i$ se define una función $f_{i}\left(X_{i}, \ldots, X_{n}, W_{i 1}, \ldots, W_{i n} \theta_{i}\right)$ que depende de los pesos de sus conexiones, del umbral y de los estados de los nodos $j$ que estén conectados con el nodo $i$. El valor de esta función proporciona el nuevo estado del nodo.

En una red neuronal artificial, los nodos se encuentran conectados por medio del contacto entre las funciones neuronales, proceso conocido como sinapsis; el comportamiento de una red neuronal artificial estará determinado, en última instancia, por la estructura de las conexiones sinápticas. A esa estructura de conexiones, se la conoce como arquitectura de la red. Se denomina arquitectura a la topología, estructura o patrón de conexiones sinápticas de una red neuronal artificial. Este tipo de redes pueden definir tanto parámetros lineales como no lineales, mapeados de una entrada a una salida, de tipo $Y=Y(X ; W, A)$, que es una función continua, tanto de las entradas como de los parámetros $W$ y de la arquitectura de red (A) (MacKay, 2003). Las redes pueden ser entrenadas para realizar tareas de regresión, clasificación y predicción.

La capa de entrada de una red neuronal artificial se define como la capa integrada por el conjunto de neuronas encargadas de recibir datos e información provenientes del entorno; este tipo de capas están constituidas por estructuras de neuronas que no cuentan con sinapsis de entrada, sino solo de salida. En segundo término, se encuentran las capas ocultas, que no tienen conexión directa con el entorno, sino que reciben información y datos de la capa de entrada; es aquí donde se tratan de estimar, modelar y representar las características del entorno. Esta capa es la única que no es de entrada ni de salida, y tiene la siguiente forma:

$$
\text { Capa oculta: } a_{j}^{1}=\sum_{l} W_{j l}^{1} X_{l}+\theta_{j}^{1} ; h_{j}=f^{1}\left(a_{j}^{1}\right)
$$

Por último, se encuentra la capa de salida, compuesta por los conjuntos de neuronas que proporcionan la respuesta de la red; este tipo de capas no tienen sinapsis de salida.

$$
\text { Capa de salida: } a_{j}^{2}=\sum_{l} W_{j l}^{2} X_{l}+\theta_{j}^{2} ; h_{j}=h^{2}\left(a_{j}^{2}\right)
$$

Una diferencia importante entre los tipos de redes neuronales artificiales que existen es el número de capas que integran los procesos de aprendizaje (capas ocultas). Un número mayor de capas permite aproximar, por lo general, procesos más complejos. Aquí se utiliza un tipo de red conocida como perceptrón multicapa. 


\section{Perceptrón multicapa}

El perceptrón multicapa es un tipo de red neuronal artificial que cuenta con una o más capas ocultas, lo que permite en principio discernir patrones más complejos, que pueden o no estar separados por más de un hiperplano. La estructura de esta red neuronal artificial puede ser la más idónea para evaluar una relación como la planteada en el presente estudio, en virtud de que permite captar un amplio rango de relaciones funcionales, incluso si estas son no lineales ${ }^{7}$. Una red de perceptrón multicapa es una red de tipo prealimentada, es decir, un tipo de red que reacciona a los cambios en su entorno para mantener algún estado concreto del sistema. Se compone de tres elementos fundamentales (Bishop, 1995):

i) Capa de entrada (variables de entrada)

ii) Capas ocultas

iii) Capas de salida (variables de salida)

El proceso mediante el cual las capas ocultas modelan las formas funcionales de los datos obtenidos en la capa de entrada se conoce como "entrenamiento de la neurona" y se lleva a cabo con un subconjunto de datos $D=\left\{X^{(n)}, t^{(n)}\right\}$, ajustando $W$ de la función de entrada y minimizando el error de la función mediante el método del "descenso del gradiente" dado por la función:

$$
E_{D}(w)=\frac{1}{2} \sum n \sum i\left(t_{i}^{(n)}-y_{i}\left(x^{(n)} ; w\right)\right)^{2}
$$

Tal minimización evalúa continuamente el gradiente de $E_{D}$ mediante la regla de la cadena para encontrar las derivadas, por medio de los siguientes pasos:

i) Se establecen los pesos sinápticos y umbrales iniciales.

ii) Se ejecuta una de las bases para obtener una respuesta de la red frente al patrón r-ésimo.

iii) Esos errores son interpretados como señales de error asociado a ese proceso; estos errores se calculan mediante:

$$
\left(\sum_{k=1}^{S}\left(\sum_{j=1}^{0} W^{\prime} k_{j} Y_{j}^{r}-\theta_{k}\right) W_{k j}\right) \frac{\partial f\left(\sum_{i=1}^{n} W_{j i} X_{j}^{r}-\theta_{j}\right)}{\partial\left(\sum_{i=1}^{n} W_{j i} X_{j}^{r}-\theta_{j}\right)}
$$

iv) Se calcula el incremento total para todos y cada uno de los patrones de los umbrales y de los pesos $\Delta W_{k j}^{\prime}$ y $\Delta W_{j i}^{\prime}$.

v) Se actualizan los pesos y los umbrales.

vi) Se vuelve a calcular el error total en $t+1$. Si ese error no es "satisfactorio", es decir, si no es el mínimo absoluto encontrado mediante el descenso del gradiente, se vuelve a interpretar el error como señal de error del proceso, y se repite el tercer paso, hasta encontrar el error mínimo.

Adicionalmente, para cada una de las capas ocultas y de salida se estima una función de activación, determinada mediante el algoritmo correspondiente de estimación. La función de activación relaciona la suma ponderada de unidades de una capa con los valores de las unidades en la capa correcta. Esta función de activación es distinta para las capas ocultas y la capa de salida. En el caso de las capas ocultas, los tipos de funciones de activación que es posible obtener son los siguientes:

\footnotetext{
7 De hecho, la estimación del modelo de redes neuronales artificiales con arquitectura de dos capas muestra mejor ajuste que el de una capa con los datos utilizados de la encuesta ENTIC. Aquí solo se reportan los resultados con mejor ajuste utilizados en el modelo. No se reportan los resultados que no fueron utilizados.
} 
i) Tangente hiperbólica: toma argumentos de los valores reales y los transforma en valores que se encuentran en un rango de $(-1,1)$, mediante la función:

$$
\gamma(c)=\tanh (c)=\frac{e^{c}-e^{-c}}{e^{c}+e^{-c}}
$$

ii) Sigmoide: toma argumentos de los valores reales y los transforma en valores que se encuentran en un rango de $(0,1)$, mediante la función:

$$
\gamma(c)=\frac{1}{\left(1+e^{-c}\right)}
$$

En el caso de las variables de salida, la función de activación relaciona la suma ponderada de unidades de una capa con los valores de unidades en la capa correcta. Es posible obtener los siguientes tipos de función de activación para la capa de salida:

i) Identidad: esta función se caracteriza por tomar argumentos de los valores reales y devolverlos sin modificarlos, mediante la función:

$$
\gamma(c)=c
$$

ii) Softmax: esta función toma un vector de argumentos de los valores reales y lo transforma en un vector cuyos elementos están entre los valores $(0,1)$ y la suma es igual a 1 . Cuando existen variables de salida que son de escala, no es posible utilizar esta función; solo está disponible en el caso de que las variables dependientes (de entrada) sean variables categóricas. Esta función tiene la forma:

$$
\gamma\left(c_{k}\right)=\exp \left(C_{k}\right) / \sum_{j} \exp \left(C_{j}\right)
$$

Una vez que la función ha logrado minimizar los errores y cuando se han encontrado las funciones de activación de las capas ocultas y de salida, el proceso se da por concluido; la manera más adecuada de contrastar los resultados obtenidos es considerar un conjunto o lote de datos por separado. Este conjunto no entra dentro de los datos de entrenamiento, por lo que representa un buen indicador de la capacidad de la red para estimar o reconocer patrones.

\section{Análisis de los resultados}

El primer paso del análisis factorial consiste en obtener los coeficientes de comunalidad de las variables analizadas; si estos coeficientes son altos (cercanos a 1) se tiene una aproximación de que un componente importante de las variables puede explicarse por la existencia de factores comunes. En caso contrario (si los coeficientes son cercanos a 0), se hace evidente que la mayor parte de la varianza del fenómeno puede ser explicada por factores únicos. Los factores se obtienen mediante el método de componentes principales.

Los coeficientes de comunalidad de la columna "extracción" representan la proporción de la varianza que puede ser explicada por el modelo de solución factorial obtenido. Las comunalidades explican la mayor parte de la varianza conjunta de las variables (véase el cuadro 1). 
Cuadro 1

Resultados del análisis factorial

\begin{tabular}{|c|c|c|c|c|c|}
\hline \multirow{3}{*}{$\begin{array}{l}\text { Componente } \\
1\end{array}$} & \multirow{3}{*}{$\begin{array}{c}\begin{array}{c}\text { Autovalores } \\
\text { iniciales }\end{array} \\
\text { Total } \\
3,684\end{array}$} & \multirow{3}{*}{$\begin{array}{c}\text { Porcentaje de varianza } \\
\text { de cada componente }\end{array}$} & \multirow{3}{*}{$\begin{array}{c}\begin{array}{c}\text { Sumas de rotación de } \\
\text { cargas al cuadrado }\end{array} \\
\text { Porcentaje acumulado } \\
24,562\end{array}$} & \multirow{2}{*}{\multicolumn{2}{|c|}{$\begin{array}{c}\text { Comunalidades } \\
\text { Comunalidades (extracción) }\end{array}$}} \\
\hline & & & & & \\
\hline & & & & Líneas fijas & 0,526 \\
\hline 2 & 2,074 & 13,824 & 38,386 & Teléfono móvil & 0,452 \\
\hline 3 & 1,259 & 8,395 & 46,781 & Redes sociales & 0,625 \\
\hline 4 & 1,002 & 6,681 & 53,462 & Computadora & 0,803 \\
\hline 5 & 0,913 & 6,090 & 59,552 & Software & 0,631 \\
\hline 6 & 0,892 & 5,948 & 65,500 & Internet & 0,807 \\
\hline 7 & 0,847 & 5,648 & 71,148 & Nube & 0,321 \\
\hline 8 & 0,824 & 5,496 & 76,644 & Innovación & 0,491 \\
\hline 9 & 0,733 & 4,886 & 81,530 & Remuneraciones de posgrado & 0,512 \\
\hline 10 & 0,646 & 4,310 & 85,840 & Remuneraciones de licenciatura & 0,671 \\
\hline 11 & 0,587 & 3,914 & 89,754 & Remuneraciones de técnico & 0,612 \\
\hline 12 & 0,542 & 3,612 & 93,366 & Capacitación en TIC & 0,671 \\
\hline 13 & 0,465 & 3,100 & 96,465 & Departamento de sistemas & 0,461 \\
\hline 14 & 0,371 & 2,470 & 98,936 & Índice de usos de Internet & 0,651 \\
\hline 15 & 0,160 & 1,064 & 100,000 & Índice de usos de software & 0,984 \\
\hline
\end{tabular}

Fuente: Elaboración propia.

Nota: Método de extracción: análisis de componentes principales. Se empleó el software SPSS 23.

El cuadro 1 muestra el conjunto de valores propios de la matriz de varianzas y covarianzas, así como el porcentaje de la varianza total que cada valor propio representa. Los autovalores obtenidos expresan la cantidad de la varianza que es explicada por cada uno de los factores. El método de extracción permite obtener tantos factores como valores propios mayores que 1 tiene la matriz analizada ( 4 en este caso). De esta forma, se extrajeron 4 componentes que permiten explicar el 53,5\% de la varianza total. El siguiente paso consiste en el cálculo de la matriz de componentes, que muestra el número de variables que satura a cada factor. Con la intención de obtener un número de variables de "estructura simple" (Thurstone, 1947), es decir, variables que saturen un único factor, y factores que contengan un número reducido de variables que se saturen inequívoca y exclusivamente en ellos, es posible rotar la matriz de componentes obtenida mediante la solución inicial ${ }^{8}$. En nuestro análisis, el método de rotación aplicado es Varimax. Los resultados de la matriz de componentes rotada se muestran en el cuadro 2.

\footnotetext{
8 Existen distintos métodos de rotación, que parten de la idea de que no existe una solución única para determinar la matriz de pesos, pudiéndose multiplicar por una matriz ortogonal de orden $k X k$, de modo que el nuevo modelo verifique las propiedades y pesos de la matriz original, pero facilitando su interpretación en términos de determinación de variables que saturan factores.
} 


\section{Cuadro 2}

Factores obtenidos con el análisis factorial

\begin{tabular}{|c|c|c|c|c|c|c|}
\hline \multirow{2}{*}{ Factor } & \multirow{2}{*}{$\begin{array}{c}\text { Porcentaje de } \\
\text { varianza explicado }\end{array}$} & \multirow{2}{*}{ Variables } & \multicolumn{4}{|c|}{ Matriz de componentes rotada ${ }^{a}$} \\
\hline & & & 1 & 2 & 3 & 4 \\
\hline \multirow{6}{*}{$\begin{array}{l}\text { 1. Infraestructura } \\
\text { de TIC }\end{array}$} & \multirow{6}{*}{24,5} & Disponibilidad de líneas fijas & 0,724 & 0,008 & $-0,034$ & 0,017 \\
\hline & & Disponibilidad de teléfono móvil & 0,377 & 0,076 & 0,308 & 0,095 \\
\hline & & Disponibilidad de computadora & 0,896 & 0,010 & 0,035 & 0,009 \\
\hline & & Disponibilidad de software & 0,469 & 0,069 & 0,032 & $-0,077$ \\
\hline & & Disponibilidad de Internet & 0,893 & 0,019 & 0,095 & 0,013 \\
\hline & & Índice de usos de Internet & 0,638 & 0,134 & 0,475 & 0,018 \\
\hline \multirow{5}{*}{$\begin{array}{l}\text { 2. Capacidades } \\
\text { de los recursos } \\
\text { humanos }\end{array}$} & \multirow{5}{*}{13,8} & Remuneraciones de posgrado & $-0,004$ & 0,698 & 0,158 & $-0,016$ \\
\hline & & Remuneraciones de licenciatura & 0,073 & 0,802 & 0,149 & 0,006 \\
\hline & & Remuneraciones de técnico & 0,047 & 0,780 & $-0,041$ & $-0,002$ \\
\hline & & Departamento de sistemas & 0,243 & 0,460 & 0,436 & 0,003 \\
\hline & & Capacitación en TIC & 0,037 & 0,573 & 0,205 & $-0,004$ \\
\hline \multirow[t]{3}{*}{ 3. Innovación } & \multirow{3}{*}{8,4} & Uso de redes sociales & 0,062 & $-0,063$ & 0,562 & 0,034 \\
\hline & & Uso de la nube & 0,028 & 0,033 & 0,564 & $-0,017$ \\
\hline & & Innovación & 0,060 & 0,170 & 0,675 & $-0,053$ \\
\hline 4. Uso de software & 6,7 & Índice de usos de software & $-0,019$ & $-0,009$ & $-0,015$ & 0,991 \\
\hline
\end{tabular}

Fuente: Elaboración propia.

Nota: Método de extracción: análisis de componentes principales. Método de rotación: Varimax con normalización Kaiser. Se empleó el software SPSS 23.

a La rotación ha convergido en cinco iteraciones.

Estos resultados indican que el conjunto de variables analizadas puede agruparse en tres factores principales, a saber, tecnología, capacidades e innovación. En otros términos, se encuentran seis variables que conforman un factor denominado "infraestructura de TIC", que explica el 25\% de la varianza conjunta, mientras que un segundo factor, compuesto por un conjunto de capacidades de los recursos humanos, explica el 14\% de la misma. Aquí, las remuneraciones según nivel de escolaridad son vistas como una aproximación de las capacidades ya que, en teoría, aquellas se relacionan con la experiencia laboral, la educación, la capacitación y las capacidades, entre otros aspectos. El uso de tecnologías de reciente aparición (las redes sociales y la nube), así como la existencia de innovación en productos o procesos, conforman un tercer factor, denominado "innovación", que explica el 8\% de la varianza conjunta. Por último, la variable de uso de software explica el 7\% de la varianza conjunta.

Una vez definidos los factores en los que colapsan las transformaciones lineales de las variables, es posible determinar si aquellos son determinantes de la productividad laboral. De acuerdo con la teoría del crecimiento endógeno, junto con los factores de capacitación de la fuerza de trabajo existen determinantes de la productividad que se relacionan con la intensidad en el uso de capital por trabajador y la capacidad de las organizaciones de absorber conocimiento del entorno competitivo en el que se encuentran (aprender haciendo). Utilizamos la inversión por empleado como variable de aproximación del acervo de capital por trabajador y, por otra parte, utilizamos las variables de años de experiencia en el mercado y la razón entre exportaciones y empleo como variables operativas del entorno competitivo.

El siguiente paso en el análisis es comprobar, mediante la metodología de redes neuronales artificiales, si las variables teóricas incluidas son capaces de explicar el comportamiento de la productividad y, en todo caso, si ese comportamiento está relacionado con las capacidades de las organizaciones o, más bien, con la tecnología. Se estima una red neuronal artificial de tipo perceptrón multicapa de 
dos capas ocultas. De esta forma, la productividad laboral está en función de las variables de la capa de entrada (obtenidas mediante el análisis factorial): i) infraestructura de TIC; ii) capacidades de los recursos humanos; iii) innovación; iv) uso de software; v) inversión por empleado; vi) exportaciones por empleado, y vii) experiencia de la empresa, medida en años. La capa de salida está constituida por la productividad laboral.

\section{Resumen de procesamiento de casos}

En total, se disponía de 6.210 observaciones, de las cuales el procedimiento excluyó 1.622 porque para cada uno de esos casos se carecía de alguna de las variables contempladas como explicativas de la productividad. La partición de la red neuronal artificial se realizó de la siguiente manera: un $78 \%$ de las observaciones para entrenamiento de la red, un 14\% para prueba y un 8\% para reserva de verificación. El proceso de entrenamiento se lleva a cabo dentro de la capa oculta y sirve para aproximar la relación funcional correcta. Sin embargo, puede ocurrir que se requiera de un número mayor de datos para el entrenamiento de la neurona, caso en el cual el lote de datos de entrenamiento utiliza datos de los segmentos de prueba y de reserva, restándolos de esos segmentos. Por su parte, el segmento de prueba sirve para validar que el proceso de aprendizaje se ha llevado a cabo de la manera adecuada, en términos de minimización del error de la función.

El segmento de la muestra destinado a la reserva no se considera dentro del entrenamiento ni dentro del proceso de validación en pruebas, sino que se utiliza para comprobar el grado de cercanía de los datos de productividad estimados por el modelo de redes neuronales artificiales con respecto a los valores reales, excluyendo la posibilidad de sesgo en la estimación. Por tratarse de datos que no fueron utilizados para el entrenamiento, constituyen un buen indicador de la capacidad de predicción del modelo, en términos de la cercanía entre los valores estimados y los reales. Después del procesamiento de la red, los casos se repartieron como se muestra en el cuadro 3.

Cuadro 3

Datos de procesamiento de la red neuronal artificial

\begin{tabular}{llrc}
\hline \multicolumn{4}{c}{ Resumen de procesamiento de casos } \\
& \multicolumn{1}{c}{ N } & Porcentaje \\
\hline \multirow{3}{*}{ Muestra } & Entrenamiento & 3556 & 78 \\
\cline { 2 - 4 } & Pruebas & 665 & 14 \\
\cline { 2 - 4 } & Reserva & 367 & 8 \\
\hline \multirow{2}{*}{ Válido } & 4588 & 100 \\
\hline Excluido & & 1622 & \\
\hline Total & 6210 & \\
\hline
\end{tabular}

Fuente: Elaboración propia.

Nota: Se empleó el software SPSS 23.

\section{Capa de entrada}

La capa de entrada está constituida por las variables independientes que, de acuerdo con una elaboración teórica, son capaces de representar el comportamiento de la variable dependiente o de capa de salida. Lo que se determina dentro de la red (capas ocultas) mediante un proceso de aprendizaje es la forma que adquiere esa representación. En el caso de la red neuronal artificial seleccionada, se incluyeron las siete variables descritas anteriormente. 


\section{Capas ocultas}

En las capas ocultas de una red neuronal artificial se estima el tipo de relación existente entre las variables de entrada y la variable dependiente. Esta estimación se realiza mediante un proceso de aprendizaje conocido como "entrenamiento de la neurona". Para que este proceso se lleve a cabo, es necesario el uso de una cantidad importante de los datos disponibles, a fin de que el entrenamiento neuronal permita aproximar la relación funcional correcta. Así, se requirió el $78 \%$ de los datos para que el proceso de aprendizaje se llevara a cabo de manera adecuada ${ }^{9}$. La arquitectura específica de la red neuronal artificial seleccionada se encuentra integrada por dos capas ocultas (número máximo de capas ocultas), cuya función de activación es una tangente hiperbólica, lo que implica que se realiza una transformación de los valores de entrada hacia valores que se encuentren en un rango de (-1 y 1), a partir de la función 12. La primera capa oculta consta de 20 unidades, mientras que la segunda consta de 15 unidades (véase el cuadro 4).

Cuadro 4

Datos de procesamiento de las capas ocultas de la red neuronal artificial

\begin{tabular}{|c|c|c|}
\hline \multicolumn{3}{|c|}{ Información de la red, capas ocultas } \\
\hline \multirow{4}{*}{ Capas ocultas } & Número de capas ocultas & 2 \\
\hline & Número de unidades en la capa oculta 1 & 20 \\
\hline & Número de unidades en la capa oculta 2 & 15 \\
\hline & Función de activación & Tangente hiperbólica \\
\hline \multicolumn{3}{|c|}{ Características de entrenamiento } \\
\hline \multicolumn{2}{|c|}{ Tasa de aprendizaje inicial } & 0,4 \\
\hline \multicolumn{2}{|c|}{ Límite inferior de la tasa de aprendizaje } & 0,001 \\
\hline \multicolumn{2}{|c|}{ Reducción de la tasa de aprendizaje, en épocas } & 10 \\
\hline \multicolumn{2}{|l|}{ Impulso } & 0,9 \\
\hline \multicolumn{2}{|c|}{ Centro del intervalo } & 0 \\
\hline \multicolumn{2}{|c|}{ Desplazamiento del intervalo } & $\pm 0,5$ \\
\hline
\end{tabular}

Fuente: Elaboración propia.

Nota: $\quad$ Se empleó el software SPSS 23.

\section{Capa de salida}

La capa de salida está constituida por la productividad laboral y -al igual que la capa de entradarequiere de una función de activación. Esta función permite comparar los resultados obtenidos con los considerados factores explicativos. En el caso de la red neuronal artificial estimada, la capa de salida es un indicador de dos aspectos:

i) Si la productividad laboral está vinculada con las variables de entrada (infraestructura y uso de TIC, capacidades de los recursos humanos, innovación, uso de software, inversión por empleado, exportaciones por empleado y experiencia de la empresa); ello se establece mediante la función de error.

ii) La importancia y el orden de importancia de cada variable independiente sobre la variable dependiente.

Lo anterior se muestra en el cuadro 5.

9 Se determina que un proceso de aprendizaje es "adecuado" cuando se encuentra que el proceso de minimización de errores (mediante el método de descenso del gradiente, explicado en la sección sobre la metodología) permite encontrar los errores mínimos de la función; en ese momento, el proceso de aprendizaje ocurrido en las capas ocultas se detiene. 
Cuadro 5

Datos de procesamiento de la capa de salida de la red neuronal artificial

\begin{tabular}{lll}
\hline \multicolumn{2}{c}{ Características de entrenamiento } \\
\hline Capa de salida & Variables independientes 1 & Productividad laboral \\
\cline { 2 - 3 } & Número de unidades & 1 \\
\cline { 2 - 3 } & Método de cambio de escala para las variables dependientes de escala & Corregido normalizado \\
\cline { 2 - 3 } & Función de activación & Tangente hiperbólica \\
\cline { 2 - 3 } & Función de error & Suma de cuadrados \\
\hline
\end{tabular}

Fuente: Elaboración propia.

Nota: Se empleó el software SPSS 23.

Además, el cuadro muestra información relacionada con las conexiones sinápticas de la capa de entrada, las capas ocultas y la capa de salida. Cada conexión puede representar una ponderación de tipo excitativo o inhibidor, lo que, en otros términos, implica el signo con el que la variable independiente actúa sobre la variable dependiente, así como la fortaleza de ese impacto. Los resultados de la estimación del modelo de red pueden analizarse en términos de la capacidad de predicción del modelo; en esta dirección, la validación final de la calidad del modelo de red se realiza comparando para la muestra reservada los valores estimados por el modelo con los valores reales, para establecer su cercanía.

Como se puede apreciar en el cuadro 6, los errores en cada uno de los procesos (en miles de pesos) son razonablemente bajos, particularmente en el caso de la muestra de reserva. Ello indica que, en promedio, para la muestra de reserva, se tiene una diferencia de poco más de 1.900 pesos (equivalentes a un error del 1,2\%) entre los datos estimados y los datos observados de productividad. La cercanía de los valores de productividad laboral estimados por el modelo de redes neuronales artificiales con respecto a los valores reales de la misma variable, para el caso de la muestra de reserva, se presenta en el gráfico 1.

Dos aspectos de interés se observan en el gráfico 1; el primero es que, en promedio, para el conjunto de la muestra reservada, el modelo de redes neuronales artificiales es capaz de predecir razonablemente el comportamiento de la productividad laboral; el segundo, para aquellas empresas cuya producción por empleado es mayor que 10 millones de pesos, el modelo tiende a subestimar los valores. Ello podría indicar que los factores que explican la productividad en las empresas de mayores ingresos son más complejos que las siete variables incorporadas en el modelo como factores determinantes de la productividad.

\section{Cuadro 6}

Datos de procesamiento (entrenamiento, prueba y reserva) de la red neuronal artificial

\begin{tabular}{|c|c|c|}
\hline \multicolumn{3}{|c|}{ Resumen del modelo } \\
\hline \multirow[t]{4}{*}{ Entrenamiento } & Error de suma de cuadrados (miles de pesos) & 2,575 \\
\hline & Error relativo (miles de pesos) & 1,006 \\
\hline & Regla de parada utilizada & $\begin{array}{l}\text { Se ha superado el tiempo máximo } \\
\text { de entrenamiento ( } 15 \text { minutos) }\end{array}$ \\
\hline & Tiempo de entrenamiento (horas) & $27: 48.7$ \\
\hline \multirow[t]{2}{*}{ Prueba } & Error de suma de cuadrados (miles de pesos) & 0,001 \\
\hline & Error relativo (miles de pesos) & 1,862 \\
\hline Reserva & Error relativo (miles de pesos) & 1,932 \\
\hline
\end{tabular}

Fuente: Elaboración propia.

Nota: Se empleó el software SPSS 23. 


\section{Gráfico 1}

Productividad laboral observada y estimada mediante el modelo de redes neuronales artificiales, muestra reservada

(En miles de pesos)

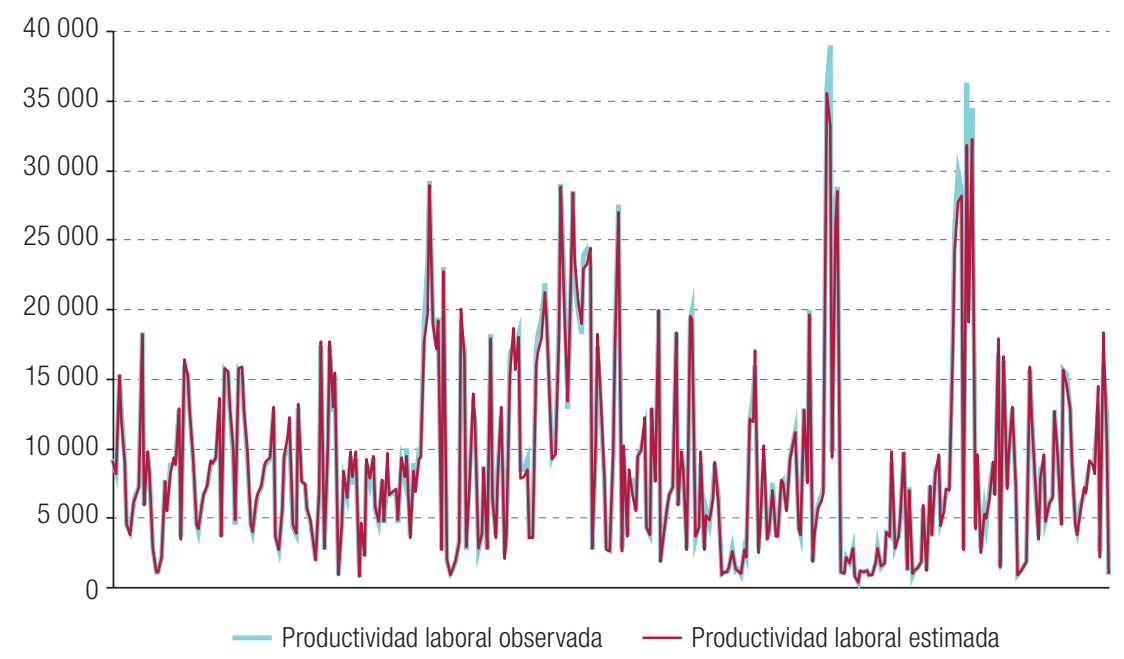

Fuente: Elaboración propia, sobre la base de datos de la Encuesta sobre Tecnologías de la Información y las Comunicaciones (ENTIC).

Nota: Estimación con una red neuronal artificial de dos capas ocultas.

Los modelos de redes neuronales artificiales no permiten observar lo que sucede dentro de las capas ocultas en términos de la obtención de coeficientes, como ocurre en el caso de los modelos econométricos; sin embargo, sí permiten obtener información relacionada con la importancia del impacto de las variables independientes sobre la variable dependiente. Ello se lleva a cabo mediante un análisis conocido como análisis de la importancia de las variables. Este análisis se muestra en el cuadro 7.

Cuadro 7

Análisis de la importancia de las variables

\begin{tabular}{lcc}
\hline Variable & Importancia & $\begin{array}{c}\text { Importancia normalizada } \\
\text { (porcentajes) }\end{array}$ \\
\hline Innovación & 0,133 & 55,9 \\
\hline Uso de software & 0,132 & 55,3 \\
\hline Disponibilidad y uso de TIC & 0,142 & 59,7 \\
\hline Capacidades de recursos humanos & 0,146 & 61,5 \\
\hline Inversión por empleado & 0,128 & 53,7 \\
\hline Exportaciones por empleado & 0,238 & 100,0 \\
\hline Antigüedad & 0,08 & 33,8 \\
\hline
\end{tabular}

Fuente: Elaboración propia.

Nota: Se empleó el software SPSS 23.

La importancia de una variable independiente es una medida que indica cuánto cambia el valor pronosticado por el modelo de la red para diferentes valores de la variable independiente. La importancia normalizada es el resultado de la división de los valores de importancia por los mayores valores de importancia expresado como porcentaje (IBM, 2011).

Los resultados del cuadro anterior muestran que para el modelo de redes neuronales artificiales estimado, la variable más relevante para entender la productividad es el volumen de exportaciones por empleado. Las capacidades educativas de la fuerza de trabajo y la disponibilidad y uso de TIC en las 
organizaciones, respectivamente, son los factores segundo y tercero en orden de importancia para explicar la productividad. La innovación (ya sea en productos o en procesos) es el factor que ocupa el cuarto lugar en términos de importancia y constituye otro de los factores que contribuyen a explicar la productividad en las empresas mexicanas de más de diez empleados; sin embargo, las empresas más innovadoras son aquellas de mayor tamaño, con porcentajes de exportaciones con respecto a los ingresos totales superiores al $20 \%$. Cuando se excluye del análisis a este grupo de empresas (menos del $4 \%$ del total de la muestra), la importancia de las exportaciones y de la innovación cae a los lugares cuarto y quinto, respectivamente, mientras que las habilidades de los recursos humanos y la disponibilidad y uso de TIC pasan a los lugares primero y segundo, en ese orden. La inversión por empleado representa en este caso el tercer factor en orden de importancia.

\section{Conclusiones}

Los estudios que analizan el impacto de las TIC sobre la productividad utilizan en su mayoría la teoría neoclásica del crecimiento como marco de referencia teórico, que atribuye al cambio tecnológico un papel central para explicar el crecimiento de la productividad. Sin embargo, esta teoría (como se demuestra en los estudios analizados aqui) no es capaz de explicar por qué las empresas que acusan niveles de adopción de TIC similares muestran productividades tan disímiles.

La mayoría de los estudios no consideran la existencia de condiciones iniciales diferenciadas, particularmente aquellas relacionadas con las capacidades de las organizaciones. El estudio aquí realizado aporta información vinculada con el hecho de que, cuando estas diferencias son consideradas en el análisis, la comprensión de las variaciones de la productividad mejora significativamente. De manera particular, ello resulta relevante para el caso de México, donde el fenómeno ha sido escasamente estudiado.

El análisis revela la existencia de cuatro factores relevantes para explicar la productividad. El primero es la disponibilidad de TIC en las empresas. Junto con esta, como segundo factor, existen un conjunto de capacidades que determinan su grado de incorporación dentro de los procesos productivos y de negocio; a este factor lo denominamos "capacidades organizacionales" y dentro de ellas se destacan la escolaridad de los trabajadores, la capacitación que reciben y la existencia de un departamento especializado en materia de TIC. Un tercer factor se vincula con el uso y aprovechamiento que se le da a la tecnología, vinculado al nivel de innovación (en productos o procesos), y el cuarto, se relaciona con el grado de intensidad de capital por trabajador.

La exposición a entornos competitivos y la experiencia en el mercado de las organizaciones determinan cierta capacidad para absorber conocimiento del entorno y elevar la productividad, como lo muestran la relevancia de las exportaciones y la antigüedad de las empresas como variables clave del nivel productivo. Cuando los factores anteriores se combinan, es factible entender las diferencias en la productividad laboral. A nivel de empresa, el análisis de redes neuronales artificiales muestra que la combinación de exportaciones, recursos humanos y uso de TIC parece explicar de manera adecuada una parte importante del comportamiento de la productividad en el caso de las organizaciones cuyos ingresos por trabajador son inferiores a 10 millones de pesos. En el caso de las empresas con productividades laborales superiores, parecen ser otros los factores que explican el comportamiento de la productividad.

El presente estudio aporta información en el sentido de que no es solo la tecnología, sino que también son factores como la educación y la madurez organizacional los que permiten dinamizar los procesos de producción y establecer los cimientos de la alta productividad. Lo anterior apunta en un sentido de complementariedad de la política pública en tres direcciones: 
i) Se debe fomentar el adecuado desarrollo de infraestructura de TIC, particularmente en aquellos sectores o actividades económicas consideradas prioritarias, ya sea porque ocupan una posición estratégica en la cadena de valor o bien por sus altos impactos en términos de encadenamientos productivos y generación de empleo. Esta política debiera estar orientada a desarrollar infraestructura de TIC suficiente y adecuada para el aprovechamiento de los sectores clave (particularmente Internet y software, base indispensable para el funcionamiento de otro tipo de servicios, como los servicios en la nube).

ii) Como ha quedado establecido en este estudio, la disponibilidad de infraestructura constituye la primera piedra, necesaria por su naturaleza, para incidir sobre la productividad laboral; sin embargo, por sí sola resulta insuficiente y las políticas gubernamentales de impulso de la infraestructura deben ir acompañadas de políticas de capacitación a los trabajadores en el uso de estas herramientas tecnológicas. Una manera de fomentar y difundir la importancia de la capacitación para el aprovechamiento adecuado de la tecnología puede ser el establecimiento de alianzas público-privadas, en las que, por ejemplo, una parte del costo de la capacitación sea cubierta con fondos provenientes de recursos públicos y la otra por la empresa, o bien el otorgamiento de algún tipo de exención fiscal para aquellas empresas que comprueben que de manera recurrente desarrollan programas de capacitación tecnológica para los empleados.

iii) Más allá del desarrollo de políticas específicas de capacitación en el aprovechamiento de herramientas vinculadas a las TIC dirigida a los empleados que actualmente laboran en las empresas, se debe establecer una política de largo plazo de desarrollo de capacidades; para lograrlo, la estrategia adecuada parece ser la misma planteada desde los primeros estudios desarrollados por la teoría del crecimiento endógeno, es decir, la creciente inversión de recursos en educación como una condición necesaria del crecimiento económico de largo plazo. En la política pública mexicana, ello pareciera estar presente en el discurso, pero dista mucho de expresarse en una política formal de inversión creciente en educación.

\section{Bibliografía}

Aghion, P. y otros (1998), Endogenous Growth Theory, Cambridge, MIT Press.

Aguilera, L., H. Cuevas-Vargas y M. González (2015), "The impact of information and communication technologies on the competitiveness: evidence of manufacturing SMEs in Aguascalientes, Mexico", International Review of Management and Business Research, vol. 4, № 3, Teherán, Universidad Islámica Azad de Damavand.

Aravena, C., C. Cavada y N. Mulder (2012), "Contribución al crecimiento económico de las tecnologías de la información y las comunicaciones y de la productividad en la Argentina, el Brasil, Chile y México", serie Estudios Estadísticos y Prospectivos, № 76 (LC/L.3439), Santiago, Comisión Económica para América Latina y el Caribe (CEPAL).

Aramendia-Muneta, M. y A. Ollo-López (2013), "ICT Impact on tourism industry", International Journal of Management Cases, vol. 15, № 2, Bosnia y Herzegovina, CIRCLE Research Centre.

Arrieta, J., J. Torres y H. Velásquez (2009), "Predicciones de modelos econométricos y redes neuronales: el caso de la acción de SURAMINV", Semestre Económico, vol.12, № 25, Medellín, Universidad de Medellín.

Balboni, M., S. Rovira y S. Vergara (eds.) (2011), ICT in Latin America: A Microdata Analysis (LC/R.2172), Santiago, Comisión Económica para América Latina y el Caribe (CEPAL).

Banco Mundial (2012), 2012 Information and Communications for Development: Maximizing Mobile, Washington, D.C.

(2011), Little Data Book on Information and Communication Technology, Washington, D.C. [en línea] http://documents.worldbank.org/curated/en/338701468335685584/pdf/635700REPLACEMOBOIS BN09780821388600.pdf. 
Basu, S. y J. G. Fernald (2006), "Information and communications technology as a general purpose technology: evidence from U.S. industry data", Working Paper, № 2006-29, San Francisco, Banco de la Reserva Federal de San Francisco.

Baumol, W., J. Panzar y R. Willing (1982), Constestable Market and the Theory of Industry Structure, Nueva York, Harcourt Brace Jovanovich.

Bayo-Moriones, A. y F. Lera-López (2007), "A firm-level analysis of determinants of ICT adoption in Spain”, Technovation, vol. 27, № 6-7, Amsterdam, Elsevier.

Bayo-Moriones, A., M. Billón y F. Lera-López (2013), "Perceived performance effects of ICT in manufacturing SMEs", Industrial Management \& Data Systems, vol. 113, № 1, Bingley, Emerald Publishing.

Bilbao-Osorio, B., S. Dutta y B. Lanvin (eds.) (2013), The Global Information Technology Report 2013: Growth and Jobs in a Hyperconnected World, Ginebra, The Business School of the World (INSEAD)/ Foro Económico Mundial.

Bishop, C. M. (1995), Neural Networks for Pattern Recognition, Oxford, Oxford University Press.

Bresnahan, T. (1999), "Computerization and wage dispersion: an analytical reinterpretation", The Economic Journal, vol. 109, № 456, Oxford, Sociedad Real de Economía.

(1997), "Testing and measurement in competition models", Advances in Economics and Econometrics: Theory and Applications. Seventh World Congress, vol. 3, D. Kreps y K. Wallis (eds.), Cambridge, Cambridge University Press.

Brynjolfsson, E. (1996), "The contribution of information technology to consumer welfare", Information Systems Research, vol. 7, №3, Catonsville, Institute for Operations Research and the Management Sciences.

_ (1993), "The productivity paradox of information technology: review and assessment", Communications of ACM, vol. 36, № 12, Nueva York, Association for Computing Machinery.

Brynjolfsson, E. y L. M. Hitt (1996), "Paradox lost? Firm-level evidence on the returns to information systems spending", Management Science, vol. 42, № 4, Catonsville, Institute for Operations Research and the Management Sciences.

Capel, L. y J. Bosch (2004), "El districte industrial de les Tecnologies de la Informació i la Comunicació (TIC) a Barcelona. Comparació amb altres ciutats europees", Scripta Nova, vol. 8, № 170, Barcelona, Universidad de Barcelona.

Carrera Portugal, A. (2010), "Las TIC como parámetro de competitividad urbana: un escenario para las economías emergentes", Biblioteca Universitaria, vol. 13, № 2, Ciudad de México, Universidad Nacional Autónoma de México (UNAM).

Cuadras, C. M. (2007), Nuevos métodos de análisis multivariante, Barcelona, CMC Editions.

Daveri, F. (2000), "Is growth an information technology story in Europe too?", IGIER Working Paper, № 168, Milán, Universidad Comercial Luigi Bocconi.

Dedrick, J., V. Gurbaxani y K. Kraemer (2003), "Information technology and economic performance: a critical review of the empirical evidence", ACM Computing Surveys, vol. 35, № 1, Nueva York, Association for Computing Machinery.

Domínguez, L. y F. Brown (2004), "Medición de las capacidades tecnológicas en la industria mexicana", Revista de la CEPAL, N 83 (LC/G.2231-P), Santiago, Comisión Económica para América Latina y el Caribe (CEPAL).

Dutta, S. y B. Bilbao-Osorio (eds.) (2012), The Global Information Technology Report 2012: Living in a Hyperconnected World, Ginebra, The Business School of the World (INSEAD)/Foro Económico Mundial.

Gatautis, R. (2008), "The impact of ICT on public and private sectors in Lithuania", Engineering Economics, vol. 59, N 4, Kaunas, Universidad Tecnológica de Kaunas.

Greenan, N., J. Mairesse y A. Topiol-Bensaid (2001), "Information technology and research and development impacts on productivity and skills: looking for correlations on French firm level data", NBER Working Paper, $N^{\circ}$ 8075, Cambridge, Oficina Nacional de Investigaciones Económicas (NBER).

Gurbaxani, V. y S. Whang (1991), "The impact of information systems on organizations and markets", Communications of the ACM, vol. 34, N 1, Nueva York, Association for Computing Machinery.

Haykin, S. (1999), Neural Networks. A Comprehensive Foundation, Singapur, Prentice-Hall.

Hollenstein, H. (2004), "Determinants of the adoption of Information and Communication Technologies (ICT): an empirical analysis based on firm-level data for the Swiss business sector", Structural Change and Economic Dynamics, vol. 15, № 3, Amsterdam, Elsevier.

IBM (2011), IBM SPSS Neural Networks 20 [en línea] https://www.spss.ch/upload/1316002997_SPSS\%20 Neural\%20Networks\%2020.pdf. 
INEGI (Instituto Nacional de Estadística y Geografía) (2013), Sistema de Cuentas Nacionales de México: productividad total de los factores 1990-2011, Ciudad de México.

Inklaar, R., M. O'Mahony y M. Timmer (2005), "ICT and Europe's productivity performance: industry-level growth account comparisons with the United States", The Review of Income and Wealth, vol. 51, № 4, Hoboken, Wiley.

Jorgenson, D. (2001), "Information technology and the U.S. economy", The American Economic Review, vol. 91, № 1, Nashville, Asociación Estadounidense de Economía.

Jorgenson, D. y K. Stiroh (2000), "Raising the speed limit: U.S. economic growth in the information age", Brookings Papers on Economic Activity, vol. 31, № 1, Washington, D.C., The Brookings Institution.

Jorgenson, D., M. Ho y K. Stiroh (2006), "Productivity growth in the new millennium and its industry origins", ponencia presentada en la "2006 Intermediate Input-output Meeting”, Sendai, 26-28 julio.

Katz, R. (2009), El papel de las TIC en el desarrollo: propuesta de América Latina a los retos económicos actuales, Barcelona, Ariel/Fundación Telefónica.

Kraemer, K. y S. Dewan (2000), "Information technology and productivity: evidence from country-level data", Management Science, vol. 46, № 4, Catonsville, Institute for Operations Research and the Management Sciences (INFORMS).

Lal, K. (2001), "The determinants of the adoption of information technology: a case study of the Indian garments industry", Information Technology, Productivity, and Economic Growth: International Evidence and Implications for Economic Development, M. Pohjola (ed.), Oxford, Oxford University Press.

Larrañaga, P., I. Inza y A. Moujahid (2003), "Modelos probabilísticos para la inteligencia artificial y la minería de datos: selección de variables" [en línea] http://leo.ugr.es/doctopgm/cartel.pdf.

Lichtenberg, F. R. (1995), "The output contributions of computer equipment and personnel: a firm level analysis", Economics of Innovation and New Technologies, vol. 3, № 3-4, Abingdon, Taylor \& Francis.

MacKay, D. J. (2003), Information Theory, Inference, and Learning Algorithms. Cambridge, Cambridge University Press.

Malone, T., J. Yates y R. Benjamin (1989), "The logic of electronic markets", Harvard Business Review, vol. 67, $N^{\circ} 3$, Boulder, Harvard University Graduate School of Business Administration.

OCDE (Organización de Cooperación y Desarrollo Económicos) (2012), Estudio de la OCDE sobre políticas y regulación de telecomunicaciones en México, París, OECD Publishing.

Peppard, J. y J. Ward (2016), The Strategic Managment of Information Systems: Building a Digital Strategy, Hoboken, Wiley.

Pitarque, A., J. F. Roy y J. C. Ruiz (1998), "Redes neuronales vs modelos estadísticos: simulaciones sobre tareas de predicción y clasificación", Psicológica, vol. 19, Varsovia, De Gruyter.

Pohjola, M. (2001), "Information technology and economic growth: a cross-country analysis", Information Technology, Productivity, and Economic Growth: International Evidence and Implications for Economic Development, Oxford, Oxford University Press.

Rivera, E. (2004), "Teorías de la regulación en la perspectiva de las políticas públicas", Gestión y Política Pública, vol. 13, № 2, Ciudad de México, Centro de Investigación y Docencia Económicas (CIDE).

Romer, P. M. (1994), "The origins of endogenous growth", The Journal of Economic Perspectives, vol. 8, № 1, Nashville, Asociación Estadounidense de Economía. (1990), "Endogenous technological change", The Journal of Political Economy, vol. 98, № 5, Chicago, The University of Chicago Press.

Schreyer, P. (2000), "The contribution of information and communication technology to output growth: a study of the G7 countries", OECD Working Paper, № 2000/02, París, OECD Publishing.

Silva, E. y A. Teixeira (2011), "Does structure influence growth? A panel data econometric assessment of 'relatively less developed' countries, 1979-2003", Industrial and Corporate Change, vol. 20, № 2, Oxford, Oxford University Press.

Spiezia, V. (2011), "Are ICT users more innovative? An analysis of ICT-enabled innovation in OECD firms", OECD Journal: Economic Studies, vol. 2011, № 1 [en línea] http://dx.doi.org/10.1787/19952856.

Solow, R. M. (1957), "Technical change and the aggregate production function", The Review of Economics and Statistics, vol. 39, № 3, Cambridge, MIT Press.

Tarute, A. y R. Gatautis (2014), "ICT impact on SMEs performance", Procedia-Social and Behavioral Sciences, vol. 110, Amsterdam, Elsevier.

Thurstone, L. L. (1947), Multiple Factor Analysis, Chicago, The University of Chicago Press.

Tödtling, F., M. Grillitsch y C. Höglinger (2012), "Knowledge sourcing and innovation in Austrian ICT CompaniesHow does geography matters", Industry and Innovation, vol. 19, № 4, Abingdon, Taylor \& Francis. 
UIT/UNESCO (Unión Internacional de Telecomunicaciones/Organización de las Naciones Unidas para la Educación, la Ciencia y la Cultura) (2013), The State of Broadband 2013: Universalizing Broadband, París. UNCTAD (Conferencia de las Naciones Unidas sobre Comercio y Desarrollo) (2003), Information and Communication Technology Development Indices, Ginebra.

Wimble, M. y H. Singh (2015), "A multilevel examination of information technology and firm performance: the interaction of industry and firm effects", PAC/S 2015 Proceedings, Pacific Asia Conference on Information Systems [en línea] https://aisel.aisnet.org/pacis2015/129. 